\section{CLINICAL PRACTICE}

Caren G. Solomon, M.D., M.P.H., Editor

\title{
Initial Management of Seizure in Adults
}

\author{
Phil E.M. Smith, M.D.
}

This Journal feature begins with a case vignette highlighting a common clinical problem. Evidence supporting various strategies is then presented, followed by a review offormal guidelines, when they exist. The article ends with the author's clinical recommendations.

An 18-year-old woman is brought to the emergency department after having had a seizure. She was up late with friends the night before and drank some alcohol. Shortly after waking this morning, she collapsed without warning, injuring her face. Her boyfriend witnessed her having a generalized tonic-clonic seizure with cyanosis during which she bit the side of her tongue. Her first memory was waking in the ambulance. She has had no previous seizures; specifically, she has not had any involuntary jerks of the arms and legs on awakening, blank spells, or sensitivity to flashing lights (e.g., sunlight flashing through trees, as seen while riding in a car). How should this patient be further evaluated and treated?

THE CLINICAL PROBLEM

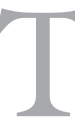

HE INCIDENCE RATE OF A SINGLE UNPROVOKED SEIZURE AMONG ADULTS is 23 to 61 cases per 100,000 person-years. ${ }^{1}$ A seizure may substantially affect a person's social interactions, employment, and driving eligibility. After a first unprovoked seizure, the overall risk of recurrence may be as high as $60 \%$ (Fig. S1 in the Supplementary Appendix, available with the full text of this article at NEJM.org), and this risk is highest within the first 2 years. ${ }^{2}$ Epilepsy affects $0.65 \%$ of adults worldwide, ${ }^{3}$ and this incidence is highest in developing countries. Epilepsy is diagnosed after two unprovoked seizures that occur more than 24 hours apart or after a single event that occurs in a person who is considered to have a high risk of recurrence ( $>60 \%$ risk in a 10 -year period). ${ }^{4}$ Abnormal findings on electroencephalography (EEG), an abnormal neurologic status, and a second seizure all increase the probability of seizure recurrence. ${ }^{5}$ These three factors allow clinicians to stratify low, medium, and high risks (Table 1) and help in guiding decisions about the initiation of antiseizure medication.

Occasionally, serial seizures or status epilepticus will manifest as a first seizure, and these conditions may be life-threatening. The management of these conditions is described elsewhere. ${ }^{6}$

STRATEGIES AND EVIDENCE

\section{DIAGNOSIS AND EVALUATION}

Expert history taking is essential in the diagnosis of an epileptic seizure. Telephoning an eyewitness is often invaluable, and home video recordings of patients with frequent seizures can help in the diagnosis. Table 2 summarizes the main differential diagnoses of a first generalized tonic-clonic seizure and provides information on the history taking, examination, and initial investigations. Careful

From the Department of Neurology, University Hospital of Wales, Cardiff, United Kingdom. Address reprint requests to Dr. Smith at the Alan Richens Epilepsy Unit, Department of Neurology, University Hospital of Wales, Heath Park, Cardiff, CF14 4XW, United Kingdom, or at smithpe@ cf.ac.uk.

N Engl J Med 2021;385:251-63. DOI: 10.1056/NEJMcp2024526 Copyright @ 2021 Massachusetts Medical Society.

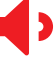

An audio version of this article is available at NEJM.org 
KEY CLINICAL POINTS

INITIAL MANAGEMENT OF SEIZURE IN ADULTS

- The clinical diagnosis of an epileptic seizure requires a detailed history taking and, ideally, an eyewitness account of the seizure.

- Evaluation with 12-lead electrocardiography is essential in a patient who has had a first seizure or an unexplained blackout spell.

- In children and teenagers, interictal electroencephalography, ideally within 24 hours after a first seizure, is particularly important.

- All patients who have had a suspected focal-onset seizure should undergo detailed magnetic resonance imaging of the head.

- Patients who have had an epileptic seizure should be informed about factors that may provoke seizures (e.g., sleep deprivation and alcohol use), the risk of a seizure occurring while driving or engaging in solitary activities, and the risks of harm from further seizures.

- Data from long-term pragmatic trials suggest that the first-line medication for patients with focal-onset seizures is either lamotrigine or levetiracetam, although other reasonable options are available; for patients with generalized-onset seizures, the first choice is sodium valproate, except for women of childbearing potential, in whom the first-line medication is usually levetiracetam.

history taking can usually distinguish the three main causes of transient loss of consciousness: epileptic seizure (provoked or unprovoked), syncope (reflex, orthostatic, or cardiac), and psychogenic nonepileptic seizure (which mimics a seizure but is caused by psychological distress rather than abnormal electrical activity in the brain).

Provoked seizures might follow transient cerebral insults such as alcohol withdrawal, the use of illicit drugs such as cocaine and methamphetamine, and metabolic disturbances (e.g., hypoglycemia or hyponatremia). They also may suggest a structural cause such as hemorrhagic stroke, encephalitis, venous sinus thrombosis, or tumor.

Seizures and epilepsy are classified according to seizure type (generalized, focal, or unknown ${ }^{8}$ ), epilepsy type, and epilepsy syndrome. ${ }^{9}$ Table 3 and Table $\mathrm{S} 1$ provide common examples of each.

The presentation of a seizure depends on its site of onset (generalized or focal) and pattern of spread. Seizures can occur at any age and in any situation. In some cases, a lack of warning suggests a generalized onset, although a lack of warning is also compatible with focal-onset seizures, especially in the frontal lobe. In other cases (usually focal-onset seizures), there is a specific but often "indescribable" aura - such as déjà vu, an epigastric "rising" sensation, or tastes or smells - usually followed by transient altered awareness.

A convulsive seizure typically has a tonic (stiffening) phase and then a clonic (convulsing) phase.
Together these phases last 1 to 3 minutes, typically while the patient has open eyes, apnea, and cyanosis. Patients awaken many minutes later feeling tired and achy, and they sometimes have a lateral tongue bite.

Physical examination may reveal findings that point to a cause other than seizure or a condition predisposing to seizure. Attention should be paid to the skin (e.g., to detect facial angiofibromas, hypomelanotic macules suggestive of tuberous sclerosis, or scars from self-harm that are often associated with psychogenic nonepileptic seizures), the cardiovascular system (an aortic ejection murmur may indicate cardiac syncope, and postural blood-pressure changes may indicate orthostatic hypotension), and findings on funduscopic examination (e.g., elevated intracranial pressure).

Basic blood tests to measure levels of electrolytes, glucose, calcium, and magnesium may help to identify potential causes of seizure or coexisting conditions. An evaluation with 12-lead electrocardiography (ECG) is indicated in all patients (especially older adults) who have had a first seizure or unexplained blackout spell to look for evidence of previous myocardial infarction because of the risk of ventricular tachycardia or of rare but potentially fatal (and often familial) disorders, including hypertrophic cardiomyopathy and long QT syndromes. ${ }^{10}$

\section{BRAIN IMAGINC}

Urgent brain imaging is warranted in patients who present with a first epileptic seizure. Com- 


\begin{tabular}{|c|c|c|c|c|c|}
\hline \multirow[t]{2}{*}{$\begin{array}{l}\text { Level of Risk and } \\
\text { No. of Seizures }\end{array}$} & \multirow[t]{2}{*}{$\begin{array}{l}\text { Neurologic Disorder } \\
\text { or Abnormal EEG }\end{array}$} & \multicolumn{3}{|c|}{ Probability of Another Seizure } & \multirow[t]{2}{*}{$\begin{array}{l}\text { Usual Recommendation } \\
\text { for Antiseizure } \\
\text { Medication }\end{array}$} \\
\hline & & By 1 yr & By 3 yr & By 5 yr & \\
\hline Low risk: 1 seizure & Neither & 0.19 & 0.28 & 0.30 & No \\
\hline \multicolumn{6}{|l|}{ Medium risk } \\
\hline 1 Seizure & Either & 0.35 & 0.50 & 0.56 & Consider \\
\hline 2-3 Seizures & Neither & 0.35 & 0.50 & 0.56 & Consider \\
\hline \multicolumn{6}{|l|}{ High risk } \\
\hline 1 seizure & Both & 0.59 & 0.67 & 0.73 & Yes \\
\hline $2-3$ seizures & Either & 0.59 & 0.67 & 0.73 & Yes \\
\hline$>3$ seizures & Neither & 0.59 & 0.67 & 0.73 & Yes \\
\hline
\end{tabular}

* Adapted from Kim et al. ${ }^{5}$ EEG denotes electroencephalogram.

puted tomography is useful and widely available. However, in most adults with a first seizure (especially a focal-onset seizure) or early epilepsy, detailed magnetic resonance imaging (MRI; ideally 3-T MRI with <3-mm slice thickness on T2-weighted imaging and fluid-attenuated inversion recovery ${ }^{11}$ ) is warranted to identify more subtle underlying causes such as hippocampal sclerosis, focal cortical dysplasia, or tumor that may be treated surgically.

\section{ELECTROENCEPHALOGRAPHY}

Interictal EEG that is performed in a patient who has had a first seizure is unlikely to capture another seizure, although the procedure may provoke psychogenic nonepileptic seizures. EEG is most informative in patients younger than 25 years of age because these patients are most likely to have subclinical interictal generalized activity that may confirm a generalized seizure tendency and that strongly predicts further seizures ( $70 \%$ positive predictive value). ${ }^{12,13}$

EEG that is performed soon after a patient has had a first seizure identifies more epileptiform abnormalities than later EEG; one study involving 300 consecutive adults and children identified abnormalities in $51 \%$ of those who underwent EEG within 24 hours and in 34\% of those who underwent EEG later. ${ }^{14}$ EEG that is performed in ambulatory or sleep-deprived patients further increases the diagnostic yield in patients in whom an epileptic seizure is likely even though the routine interictal EEG findings are normal. ${ }^{15}$ The presence of interictal epileptiform discharges in either of these investigations increases the 1-year risk of seizure recurrence by a factor of $1.5 .{ }^{16}$

\section{MA NAGE MENT}

\section{ANTISEIZURE MEDICATIONS}

The medical management of epilepsy predominantly involves seizure suppression with the longterm use of oral medication (Table 4 and Table S2). Antiseizure medication is primarily indicated when the risk of further spontaneous seizures is judged to exceed $60 \%$ over the next 10 years.

The aim of management is no seizures and minimal adverse effects of treatment. However, if these goals prove to be impossible, then the priority is complete control of major convulsive seizures, which are potentially dangerous because they may increase the risk of sudden unexpected death in epilepsy (SUDEP) above the estimated absolute risk among patients with epilepsy overall (1.2 cases per 1000 patient-years). ${ }^{23}$

The initiation of long-term use of antiseizure medication is a major decision that is made by the patient and the clinician. This decision requires reasonable certainty of an epilepsy diagnosis; the use of medication for a trial period in patients in whom the diagnosis is uncertain should be avoided.

The Medical Research Council Multicentre Trial for Early Epilepsy and Single Seizures ${ }^{24}$ showed that the risk of seizure recurrence was 


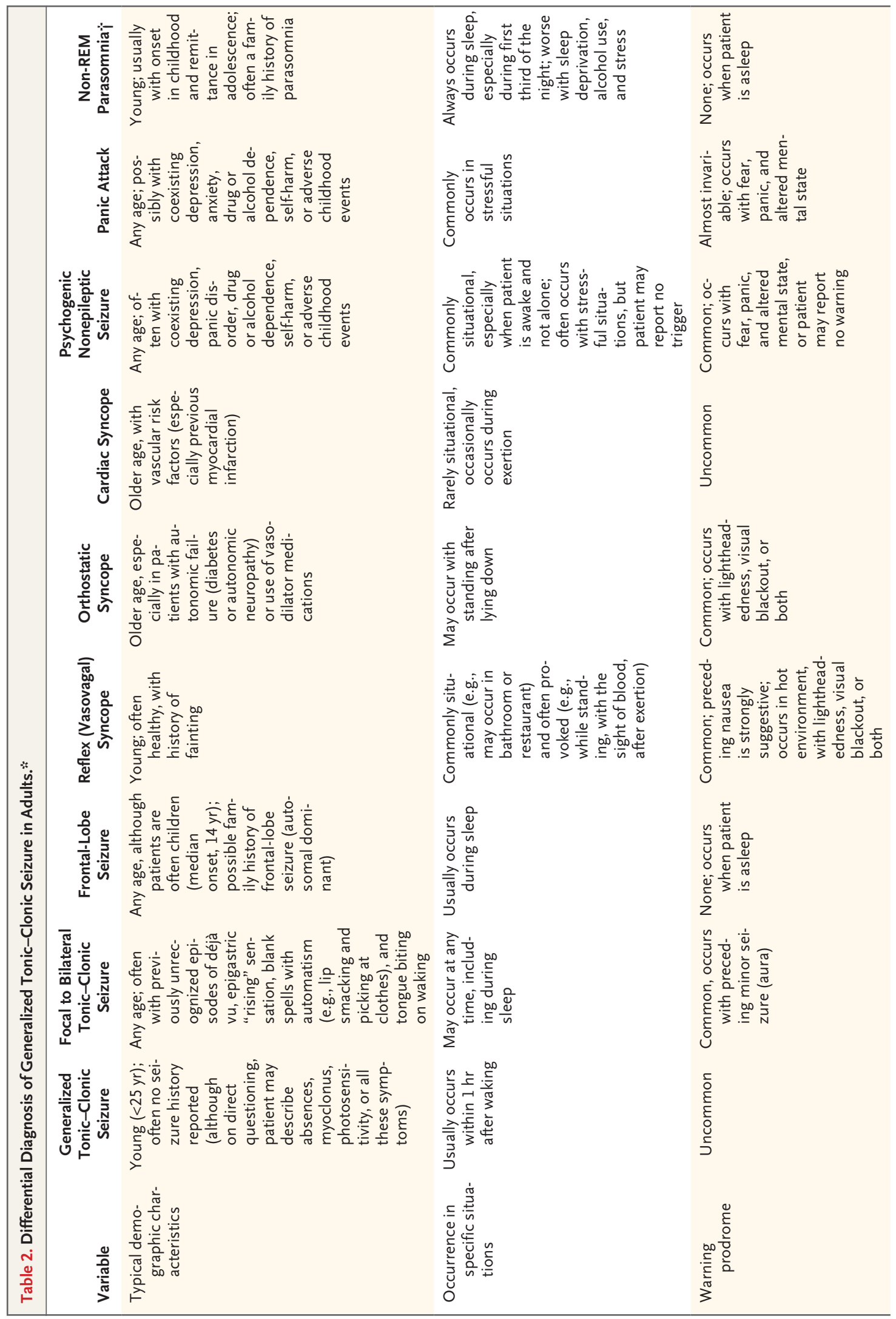




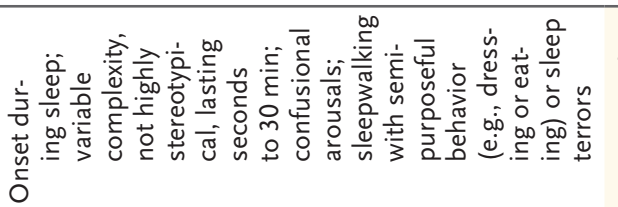

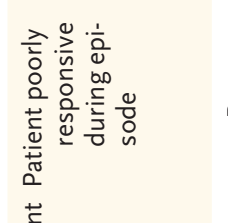

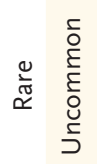

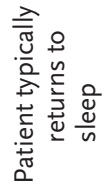

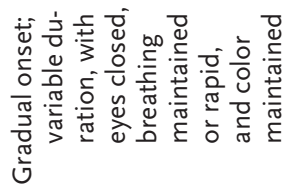

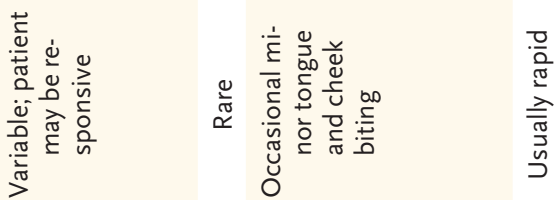

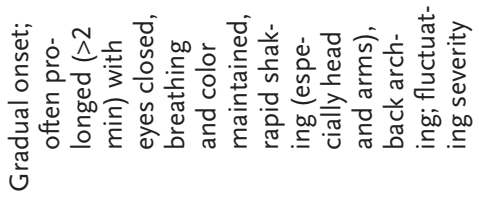

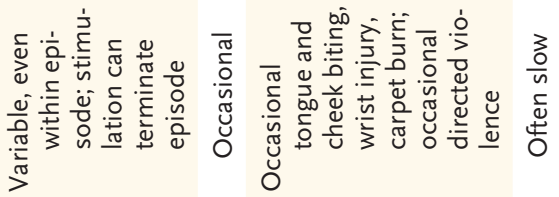

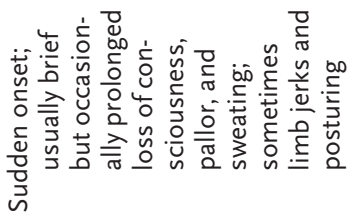

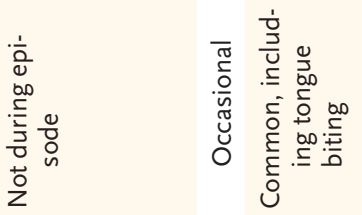

$\frac{0}{2}$
$\frac{0}{0}$
$\frac{5}{4}$
0
0

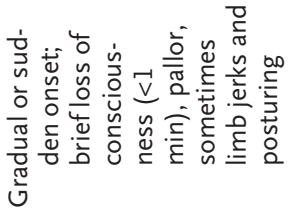

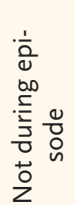

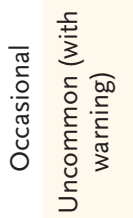

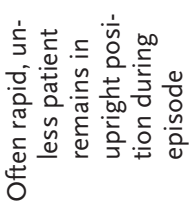

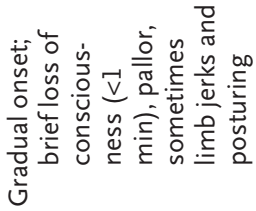
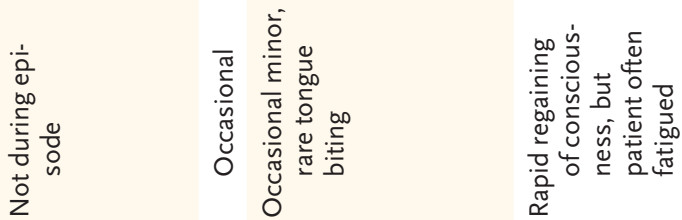

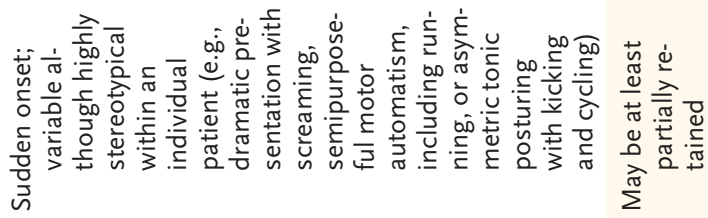

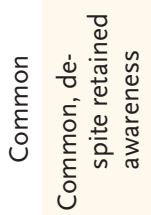

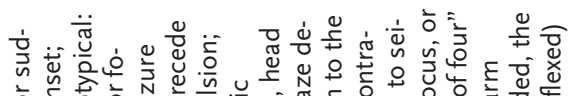

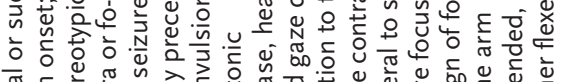

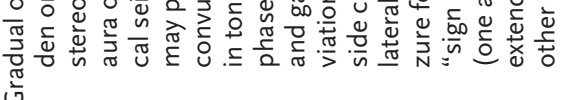
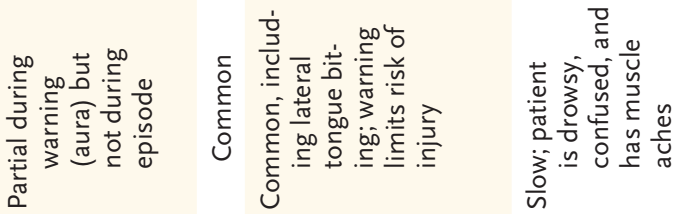

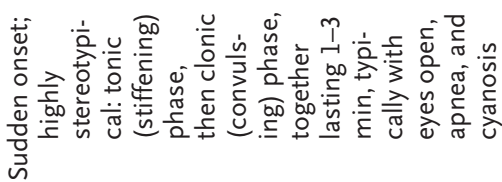
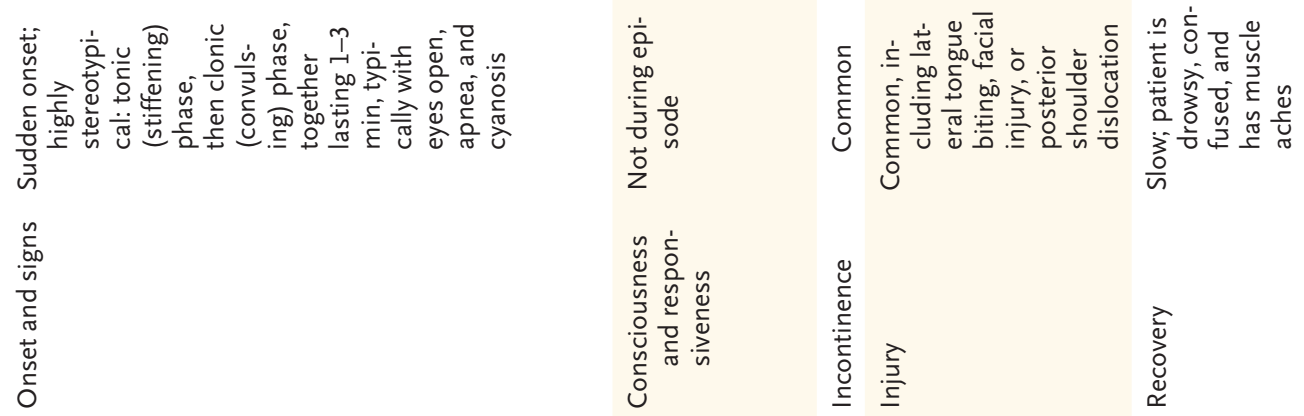

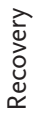




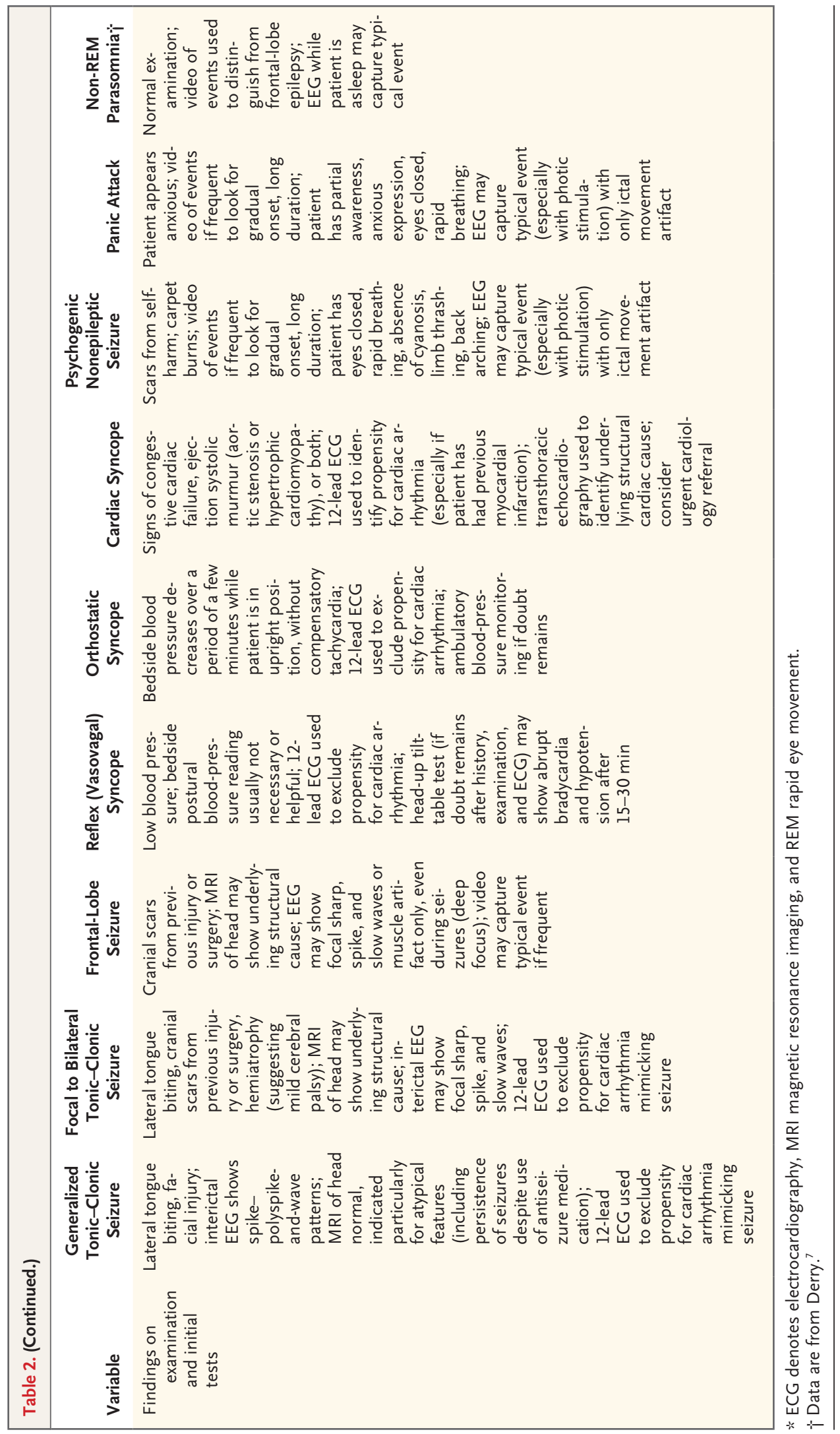


Table 3. Common Types of Seizures in Adolescents and Adults."

Seizure Type

Generalized onset

Motor

Nonmotor

Focal onset

Awareness

Motor features

Secondary generalization

Unknown onset

\section{Description and Common Examples}

The patient's symptoms or description of the seizure by a witness do not indicate an anatomical localization of the seizure. It is thought to start within and rapidly engage bilaterally distributed cerebral networks.

Myoclonic seizures manifest as involuntary "jumps" of the arms, legs, or head, especially shortly after waking and with sleep deprivation; generalized tonic-clonic seizures typically occur without warning, although they may follow myoclonic or absence seizures and are most likely to occur within $1 \mathrm{hr}$ after waking and with sleep deprivation.

Typical absences manifest as a brief loss of awareness, with an abrupt onset and offset, provoked by hyperventilation, often with eyelid flickering, and ictal 3-Hz generalized spike-and-wave activity on EEG; atypical absences have a less abrupt onset and offset, with an atypical, generalized spikeand-wave activity on EEG that is slower $(<2.5 \mathrm{~Hz})$ than that in typical seizures.

Most new-onset seizures in adults, including tonic-clonic seizures, are of focal onset. There is clinical evidence of seizure onset localized to one part of the brain, regardless of whether it subsequently involves the remainder of the brain. The site of onset determines the features: temporal lobe (epigastric "rising" sensation, déjà vu, and smell or taste), frontal lobe (features are often sleep-related, with adversive head turn, arm and leg jerking, and speech arrest), occipital lobe (elementary visual hallucinations in the contralateral visual field), parietal lobe (lateralized sensory symptoms, including pain), or insular cortex (laryngeal constriction, dyspnea, and contralateral somatosensory symptoms).

In focal-onset aware (formerly called simple partial) seizures, awareness of the self or environment is retained; in focal-onset impaired awareness (formerly called complex partial) seizures, awareness of the self or environment is impaired.

Motor seizures include automatisms (e.g., lip smacking and picking at clothes) and atonic, tonic, clonic, and myoclonic features; nonmotor seizures include autonomic, behavior arrest, cognitive, emotional, and sensory features.

In focal to bilateral tonic-clonic (formerly called secondarily generalized) seizures, the focal seizure develops into a tonic-clonic seizure. Such seizures often first occur during sleep.

The origin of a seizure is often uncertain, especially after only one seizure.

* Data are from Fisher et al. ${ }^{8}$

lower in the first 2 years after the first seizure among patients who received immediate initiation of medication (generally carbamazepine or sodium valproate) than among those who received delayed treatment pending a second seizure (32\% vs. $39 \%$ ), but earlier initiation of treatment did not affect longer-term seizure remission. Adverse events were significantly more common with immediate treatment than with delayed treatment (in 39\% and 31\% of the patients), and quality-of-life measures were similar in the two groups. Therefore, clinicians usually advise withholding medication in patients who have had a single seizure unless the recurrence risk is particularly high. ${ }^{4}$ Despite a low estimated risk of recurrence, some patients choose to receive med- ication because they have had a particularly severe or injurious first seizure or because they live in areas such as the United Kingdom where a second seizure might extend the driving restriction from 6 months to 12 months.

\section{FACTORS GUIDING MEDICATION CHOICE}

The choice of medication should be guided by the type of seizure and epilepsy syndrome (broadly, valproate or levetiracetam is used in patients with generalized-onset seizures and lamotrigine or levetiracetam is used in those with focal-onset seizures) as well as by the effectiveness, adverseevent profile, and pharmacodynamic and pharmacokinetic properties of a given drug. Coexisting conditions must also be considered. For example, 


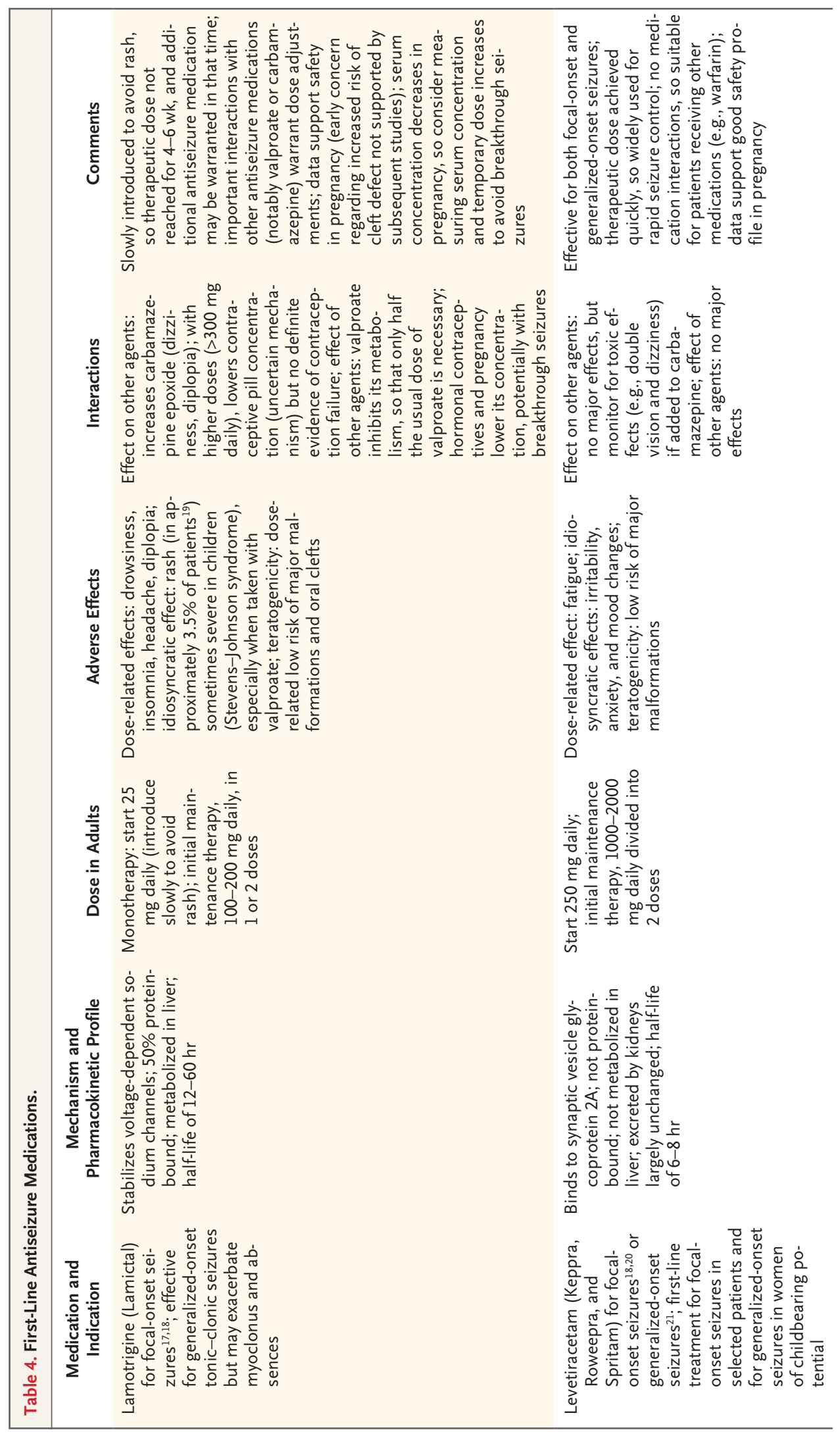



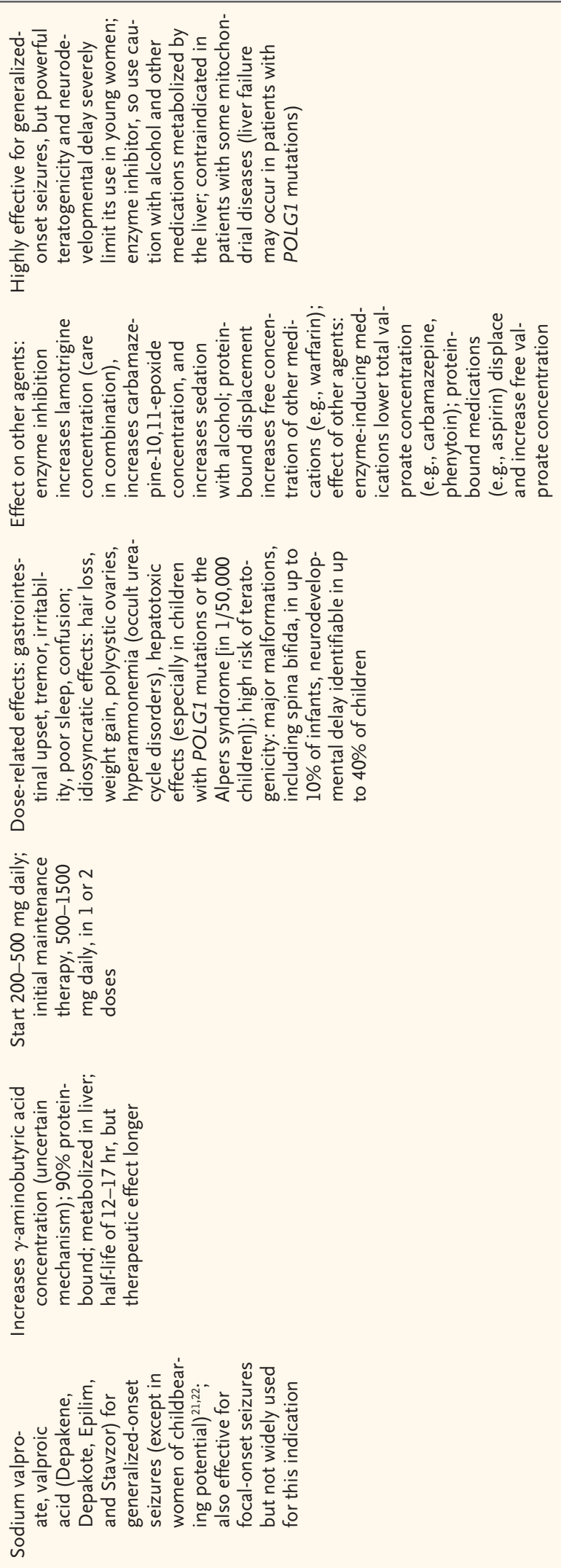

patients with substantial anxiety may prefer lamotrigine over levetiracetam, whereas those with obesity or migraines may choose topiramate, which can suppress appetite and reduce the incidence of headaches. An overriding consideration for women is the effects of medication on potential pregnancy.

Although a detailed discussion of the use of antiseizure medication in women who may become pregnant is beyond the scope of this article, sodium valproate carries high risks in pregnancy. Approximately $10 \%$ of babies exposed to sodium valproate in utero have major congenital anomalies, ${ }^{25}$ and up to $40 \%$ have measurable neurodevelopmental delay. ${ }^{26}$ In the European Registry of Antiepileptic Drugs and Pregnancy (EURAP) Study Group prospective study involving 7555 pregnancies, ${ }^{27} 10.3 \%$ of the infants had major congenital malformations after in utero exposure to valproate, $5.5 \%$ had these malformations after exposure to carbamazepine, $3.9 \%$ after topiramate, $3.0 \%$ after oxcarbazepine, $2.9 \%$ after lamotrigine, and $2.8 \%$ after levetiracetam (as compared with a $2.6 \%$ risk among infants who had not been exposed in utero to antiseizure medica$\operatorname{tion}^{28}$ ). The possible contribution of maternal seizures to the risks of congenital anomalies and neurodevelopmental delay remains unclear.

The EURAP study also showed that major congenital malformations associated with valproate were dose-related and included cardiac defects and hypospadias, each of which was found in $2 \%$ of infants with exposure to valproate; cleft lip; gastrointestinal, renal, and neural-tube defects; and polydactyly. Cognitive assessments in 6-year-old children who had had in utero exposure to valproate showed significant dose-related inverse associations with IQ, verbal ability, and nonverbal ability; these effects were not observed in children with in utero exposure to other antiseizure medications. ${ }^{26}$ Thus, valproate should generally be avoided in women of childbearing potential; if valproate is used, effective measures should be taken to prevent pregnancy unless the woman is fully informed about the risks. As part of a licensing requirement since 2018 in the United Kingdom and the European Union, women who receive valproate must use highly reliable contraception (a hormonal implant or an intrauterine device) or undergo monthly pregnancy tests, and they must sign an annual risk-acknowledgment form. ${ }^{29}$ 
Data from pregnancy registries have shown no consistent safety signals for lamotrigine or levetiracetam $^{30}$ and no clear evidence of neurodevelopmental delay associated with these agents. ${ }^{31}$ In observational studies, maternal folate supplementation has been associated with a reduced risk of neurocognitive abnormalities among babies with in utero exposure to antiseizure medications, ${ }^{32}$ and such supplements are routinely recommended in women who may become pregnant while receiving such medication.

\section{EFFECTIVENESS OF MEDICATIONS}

A single-center observational study involving 525 patients with epilepsy of various types showed that approximately half became seizure-free for at least 1 year after they began to receive a first antiseizure medication. ${ }^{33}$ Many randomized, controlled trials of the efficacy of new antiseizure medications have assessed their use as add-on medications in patients with treatment-resistant epilepsy. In these short-term trials, these new medications reduced the frequency of seizures 2 to 4 times more than placebo ${ }^{34}$ but often at doses that were higher than those generally used in practice.

The management of epilepsy, which is a longterm condition, is largely informed by the Standard and New Antiepileptic Drugs (SANAD) trials, which involved long-term, head-to-head, unblinded comparisons of existing standard agents with newer medications. The first SANAD trial involving patients with generalized and unclassified epilepsies compared valproate (then the standard of care) with lamotrigine or topiramate and showed the superiority of valproate over topiramate with respect to treatment failure and the superiority over lamotrigine with respect to 12 -month remission. ${ }^{22}$ For focal epilepsies, lamotrigine was superior to carbamazepine (then the standard of care), gabapentin, and topiramate with respect to treatment failure and was noninferior to carbamazepine with respect to 12-month remission. ${ }^{17}$ More recently, the SANAD II trial involving patients with generalized and unclassified epilepsies did not show noninferiority of levetiracetam to valproate with respect to 12-month remission; valproate resulted in a higher incidence of 12-month remission (36\% vs. $26 \%$ and a similar incidence of adverse events, and it was more cost-effective. ${ }^{21}$ For focal epilepsies, zonisamide but not levetiracetam was non- inferior to lamotrigine with respect to 12-month remission; however, as compared with both levetiracetam and zonisamide, lamotrigine resulted in lower incidences of treatment failure and adverse events, and it was more cost-effective. ${ }^{18}$

Thus, the first-line medication for patients with generalized-onset seizures is sodium valproate, or levetiracetam for girls and women of childbearing potential. For patients with focalonset seizures, lamotrigine is usually the firstline medication, although levetiracetam or other agents may have advantages in some patients (Table 4 and Fig. S2).

The main disadvantage of lamotrigine is its low starting dose, with increases to the full treatment dose over a period of several weeks. This gradual dose adjustment is necessary to reduce the risk of the Stevens-Johnson syndrome and toxic epidermal necrolysis (from $1.0 \%$ to approximately 0.01 to $0.10 \%)^{35}$; initial coverage with another antiseizure medication may be warranted. The main adverse effects of levetiracetam are irritability and anxiety, especially in patients with preexisting anxiety.

\section{LIFESTYLE FACTORS}

Clinicians should engage in joint decision making with patients and share verbal and written information. Information on driving eligibility is particularly important. In the United Kingdom and the European Union, a 6-month driving restriction is mandated for patients who have had a single seizure with a low risk of recurrence, and a 12-month restriction is mandated for patients with epilepsy, including those who have had a single seizure and who have a high risk of recurrence (e.g., those with an abnormal EEG, neurologic deficit, or both). In the United States, eligibility for a driver's license in persons who have had a single seizure or in those with epilepsy varies among states, ${ }^{36}$ although the rules are generally less restrictive than those in Europe.

Advice from clinicians regarding other activities depends on the characteristics and frequency of the patient's seizures; these factors are balanced against individual priorities. Clinicians should inform patients of the risks associated with seizures, including drowning and SUDEP; the likelihood of seizure recurrence (Table 1); and suggested lifestyle modifications (e.g., avoiding being alone during certain activities such as caring for children or bathing, so that another 
person can help if a seizure occurs, and appreciating the risks of ladders and heights).

Patients should be encouraged to adhere to the regimen of antiseizure medication and a regular sleep schedule and to limit the use of alcohol. Considerable observational data provide support for a relationship between insufficient sleep and seizure risk or abnormal EEG activity. ${ }^{37}$ A short-term randomized trial ${ }^{38}$ involving 84 patients with medication-resistant focal epilepsy in whom the dose of antiseizure medication was being tapered showed no significant differences in seizure frequency between the group of patients with sleep deprivation and the control group. However, these trial findings may not be applicable to patients with early epilepsy, and the promotion of sleep hygiene in patients with epilepsy remains prudent. Alcohol use is an important seizure precipitant, mainly because of the risk of seizure during alcohol withdrawal and the tendency of alcohol to disrupt sleep, interfere with adherence to antiseizure medications, or both. A meta-analysis of observational studies showed a dose-response relationship between the amount of alcohol consumed daily and the probability of development of epilepsy; for an average of 4, 6, and 8 drinks daily, the relative risks were 1.81 (95\% confidence interval [CI], 1.59 to 2.07), 2.44 (95\% CI, 2.00 to 2.97), and 3.27 (95\% CI, 2.52 to 4.26 ). ${ }^{39}$ Alcohol abstinence is probably unnecessary, but consumption should be limited to modest amounts. Illicit drugs that disrupt sleep, especially cocaine and amphetamine, should be avoided, but highquality data on the recreational use of cannabis in persons with epilepsy are lacking.

AREAS OF UNCERTAINTY

The clinical diagnosis of epilepsy may be incorrect in up to $20 \%$ of patients ${ }^{40}$ unless episodes are captured on EEG with video. Many patients with a diagnosis of epilepsy are later recognized to have psychogenic seizures, and additional psychogenic seizures may later develop in persons with established epilepsy. Clinicians must repeatedly question the diagnosis in patients with medication-resistant epilepsy.

The potential long-term effects of new antiseizure medications, which are typically prescribed as lifelong treatments, warrant further study. Notoriously, for 8 years after licensing, vigabatrin was used worldwide to manage seizures until it was recognized that long-term use of this agent caused permanent visual-field defects in more than half of patients. ${ }^{41}$ Data are lacking to inform pregnancy and offspring outcomes associated with new antiseizure medications; several worldwide pregnancy registries regularly update clinicians on the teratogenicity of these agents (Table S3). ${ }^{30}$

Genetic characterization has enabled both targeting of more effective treatments for some complex epilepsies (e.g., stiripentol for the Dravet syndrome $e^{42}$ and a ketogenic diet for glucose transporter type 1 deficiency syndrome $e^{43}$ ) and screening for the HLA-B` 1502 allele in Han Chinese populations to predict the carbamazepineinduced Stevens-Johnson syndrome. ${ }^{44}$ Further understanding of the effect of genetic factors on the risk of recurrent seizures and on the efficacy and risks of various medications is needed to guide treatment decisions.

\section{G U I DE L I N E S}

In 2015, the American Academy of Neurology and the American Epilepsy Society provided joint guidelines on the management of unprovoked first seizure in adults. ${ }^{2}$ The 2012 guidelines ${ }^{45}$ of the National Institute for Health and Care Excellence in the United Kingdom are undergoing revision. The current recommendations differ from these older guidelines with respect to specific medications recommended, since the results of the SANAD II trial were published after these guidelines were issued.

CONCLUSIONS AND

RECOMMENDATIONS

In the patient described in the vignette, the first generalized tonic-clonic seizure developed after sleep loss and alcohol use. Careful questioning revealed that this was an isolated event, with no previous myoclonic jerks or absences. Evaluation should include MRI of the head, interictal EEG, and 12-lead ECG. I would discuss with the patient lifestyle factors such as the importance of regular sleep and limiting alcohol consumption, the risks associated with seizures (including drowning and SUDEP), and driving eligibility. Antiseizure medications are not routinely recommended for patients who have had a single seizure; 
however, if interictal EEG showed spike-andwave activity, indicating a high risk of recurrent seizure, I would recommend initiation of an antiseizure medication. Provided that this patient did not have depression or anxiety, I would favor levetiracetam administered with a folate supplement since the patient is of childbearing potential. I would arrange follow-up in 2 months to review the patient's response and adherence to the medication regimen and any adverse effects.

No potential conflict of interest relevant to this article was reported.

Disclosure forms provided by the author are available with the full text of this article at NEJM.org.
REFERENCES

1. Hauser WA, Beghi E. First seizure definitions and worldwide incidence and mortality. Epilepsia 2008;49:Suppl 1:8-12. 2. Krumholz A, Wiebe S, Gronseth GS, et al. Evidence-based guideline: management of an unprovoked first seizure in adults: report of the guideline development subcommittee of the American Academy of Neurology and the American Epilepsy Society. Neurology 2015;84:1705-13. 3. Fiest KM, Sauro KM, Wiebe S, et al. Prevalence and incidence of epilepsy: a systematic review and meta-analysis of international studies. Neurology 2017;88: 296-303.

4. Fisher RS, Acevedo C, Arzimanoglou A, et al. ILAE official report: a practical clinical definition of epilepsy. Epilepsia 2014;55:475-82

5. Kim LG, Johnson TL, Marson AG, Chadwick DW. Prediction of risk of seizure recurrence after a single seizure and early epilepsy: further results from the MESS trial. Lancet Neurol 2006;5:317-22. 6. Jones S, Pahl C, Trinka E, Nashef L. A protocol for the inhospital emergency drug management of convulsive status epilepticus in adults. Pract Neurol 2014; 14:194-7.

7. Derry CP. Sleeping in fits and starts: a practical guide to distinguishing nocturnal epilepsy from sleep disorders. Pract Neurol 2014;14:391-8.

8. Fisher RS, Cross JH, French JA, et al. Operational classification of seizure types by the International League Against Epilepsy: position paper of the ILAE Commission for Classification and Terminology. Epilepsia 2017;58:522-30.

9. Scheffer IE, Berkovic S, Capovilla G, et al. ILAE classification of the epilepsies: position paper of the ILAE Commission for Classification and Terminology. Epilepsia 2017;58:512-21.

10. Brugada P, Geelen P. Some electrocardiographic patterns predicting sudden cardiac death that every doctor should recognize. Acta Cardiol 1997;52:473-84.

11. Duncan JS. Brain imaging in epilepsy. Pract Neurol 2019;19:438-43.

12. Collins S, Iansek R. A prospective study of the predictive value of electroencephalographic abnormalities for epileptic loss of consciousness. Clin Exp Neurol 1988;25:103-8.

13. Hauser WA, Rich SS, Annegers JF, An- derson VE. Seizure recurrence after a 1st unprovoked seizure: an extended followup. Neurology 1990;40:1163-70.

14. King MA, Newton MR, Jackson GD, et al. Epileptology of the first-seizure presentation: a clinical, electroencephalographic, and magnetic resonance imaging study of 300 consecutive patients. Lancet 1998;352:1007-11.

15. Geut I, Weenink S, Knottnerus ILH van Putten MJAM. Detecting interictal discharges in first seizure patients: ambulatory EEG or EEG after sleep deprivation? Seizure 2017;51:52-4.

16. Koutroumanidis M, Bruno E. Epileptology of the first tonic-clonic seizure in adults and prediction of seizure recurrence. Epileptic Disord 2018;20:490-501. 17. Marson AG, Al-Kharusi AM, Alwaidh $M$, et al. The SANAD study of effectiveness of carbamazepine, gabapentin, lamotrigine, oxcarbazepine, or topiramate for treatment of partial epilepsy: an unblinded randomised controlled trial. Lancet 2007 369:1000-15.

18. Marson A, Burnside G, Appleton R et al. The SANAD II study of the effectiveness and cost-effectiveness of levetiracetam, zonisamide, or lamotrigine for newly diagnosed focal epilepsy: an open-label, non-inferiority, multicentre, phase 4 , randomised controlled trial. Lancet 2021 397:1363-74.

19. Mani R, Monteleone C, Schalock PC Truong T, Zhang XB, Wagner ML. Rashes and other hypersensitivity reactions associated with antiepileptic drugs: a review of current literature. Seizure 2019;71:270-8. 20. Brodie MJ, Perucca E, Ryvlin P, BenMenachem E, Meencke HJ; Levetiracetam Monotherapy Study Group. Comparison of levetiracetam and controlled-release carbamazepine in newly diagnosed epilepsy. Neurology 2007;68:402-8.

21. Marson A, Burnside G, Appleton R et al. The SANAD II study of the effectiveness and cost-effectiveness of valproate versus levetiracetam for newly diagnosed generalised and unclassifiable epilepsy: an open-label, non-inferiority, multicentre, phase 4 , randomised controlled trial. Lancet 2021;397:1375-86.

22. Marson AG, Al-Kharusi AM, Alwaidh $M$, et al. The SANAD study of effectiveness of valproate, lamotrigine, or topiramate for generalised and unclassifiable epilep- sy: an unblinded randomised controlled trial. Lancet 2007;369:1016-26.

23. Sveinsson O, Andersson T, Mattsson P, Carlsson S, Tomson T. Clinical risk factors in SUDEP: a nationwide populationbased case-control study. Neurology 2020; 94(4):e419-e429.

24. Marson A, Jacoby A, Johnson A, et al. Immediate versus deferred antiepileptic drug treatment for early epilepsy and single seizures: a randomised controlled trial. Lancet 2005;365:2007-13.

25. Weston J, Bromley R, Jackson CF, et al. Monotherapy treatment of epilepsy in pregnancy: congenital malformation outcomes in the child. Cochrane Database Syst Rev 2016;11:CD010224.

26. Meador KJ, Baker GA, Browning N, et al. Fetal antiepileptic drug exposure and cognitive outcomes at age 6 years (NEAD study): a prospective observational study. Lancet Neurol 2013;12:244-52.

27. Tomson $\mathrm{T}$, Battino $\mathrm{D}$, Bonizzoni $\mathrm{E}$, et al. Comparative risk of major congenital malformations with eight different antiepileptic drugs: a prospective cohort study of the EURAP registry. Lancet Neurol 2018;17:530-8

28. Veroniki AA, Cogo E, Rios P, et al. Comparative safety of anti-epileptic drugs during pregnancy: a systematic review and network meta-analysis of congenital malformations and prenatal outcomes. BMC Med 2017;15:95.

29. New measures to avoid valproate exposure in pregnancy endorsed. European Medicines Agency. July 6, 2018 (https:// www.ema.europa.eu/en/medicines/human/ referrals/valproate-related-substances-0). 30. Tomson T, Battino D, Craig J, et al. Pregnancy registries: differences, similarities, and possible harmonization. Epilepsia 2010;51:909-15.

31. Baker GA, Bromley RL, Briggs M, et al. IQ at 6 years after in utero exposure to antiepileptic drugs: a controlled cohort study. Neurology 2015;84:382-90.

32. Meador KJ, Pennell PB, May RC, et al. Effects of periconceptional folate on cognition in children of women with epilepsy: NEAD study. Neurology 2020;94(7): e729-e740.

33. Kwan P, Brodie MJ. Early identification of refractory epilepsy. N Engl J Med 2000;342:314-9.

34. Marson AG, Kadir ZA, Chadwick DW. 
New antiepileptic drugs: a systematic review of their efficacy and tolerability. BMJ 1996;313:1169-74.

35. Mockenhaupt M, Messenheimer J, Tennis P, Schlingmann J. Risk of StevensJohnson syndrome and toxic epidermal necrolysis in new users of antiepileptics. Neurology 2005;64:1134-8.

36. State driving laws database. Epilepsy Foundation (https://www.epilepsy.com/ driving-laws/2008801/2008731)

37. Rossi KC, Joe J, Makhija M, Goldenholz DM. Insufficient sleep, electroencephalogram activation, and seizure risk re-evaluating the evidence. Ann Neurol 2020;87:798-806.

38. Malow BA, Passaro E, Milling C
Minecan DN, Levy K. Sleep deprivation does not affect seizure frequency during inpatient video-EEG monitoring. Neurology 2002;59:1371-4

39. Samokhvalov AV, Irving H, Mohapatra S, Rehm J. Alcohol consumption, unprovoked seizures, and epilepsy: a systematic review and meta-analysis. Epilepsia 2010; 51:1177-84.

40. Chadwick D, Smith D. The misdiagnosis of epilepsy. BMJ 2002;324:495-6.

41. Maguire MJ, Hemming K, Wild JM, Hutton JL, Marson AG. Prevalence of visual field loss following exposure to vigabatrin therapy: a systematic review. Epilepsia 2010;51:2423-31.
Dravet syndrome. Drugs 2019;79:178596.

43. Kass HR, Winesett SP, Bessone SK, Turner Z, Kossoff EH. Use of dietary therapies amongst patients with GLUT1 deficiency syndrome. Seizure 2016;35:83-7.

44. Ferrell PB Jr, McLeod HL. Carbamazepine, HLA-B ${ }^{\star} 1502$ and risk of StevensJohnson syndrome and toxic epidermal necrolysis: US FDA recommendations. Pharmacogenomics 2008;9:1543-6.

45. Epilepsies: diagnosis and management clinical guideline. National Institute for Health and Care Excellence. January 11, 2012 (www.nice.org.uk/guidance/ cg137).

Copyright (C) 2021 Massachusetts Medical Society.

The Journal welcomes consideration of new submissions for Images in Clinical Medicine. Instructions for authors and procedures for submissions can be found on the Journal's website at NEJM.org. At the discretion of the editor, images that are accepted for publication may appear in the print version of the Journal, the electronic version, or both. 